\section{Review: compression was effective for healing venous ulcers, and multicomponent systems were better than single-component ones}

\section{QUESTIONS}

Do compression bandages or stockings help venous ulcer healing? Which compression bandage or stocking is most effective?

\section{REVIEW SCOPE}

Included studies were randomised controlled trials that assessed compression bandages or stockings for treating venous ulcers. Outcome was objectively measured ulcer healing.

\section{REVIEW METHODS}

Medline, CINAHL, EMBASE/Excerpta Medica, Cochrane Wounds Group Specialised Register, and Cochrane Central Register of Controlled Trials (all to Oct 2008); and reference lists were searched. Experts and pharmaceutical companies were contacted. 39 RCTs (47 comparisons) were included.

\section{MAIN RESULTS}

Compression v no compression (7 RCTs). 1 of 2 RCTs showed no benefit for compression over primary dressings; 1 RCT did not report complete healing. More patients had complete healing at 12 months with compression than with non-compressive bandages (1 RCT; relative benefit increase [RBI] 130\%, 95\% CI $43 \%$ to $341 \%$; number needed to treat [NNT] 2, CI 2 to 4 ). 4 RCTs compared compression with usual care; 3 found a benefit for complete healing (1 RCT, RBI 300\%, CI 54\% to 1085\%; NNT 2, CI 2 to 6; 1 RCT, 71\% v 25\%, p not reported) or time to healing (1 RCT). Single-component (SC) compression systems (6 RCTs). 1 RCT showed no difference between SC inelastic compression and 4-layer bandages (4LB). 5 RCTs compared SC elastic compression with other types of compression; 1 showed more patients with complete healing at 24 weeks in the 4LB group than in the SC bandage group (RBI 35\%, CI 9\% to 69\%; NNT 6, CI 4 to 20). 2-component compression systems (3 RCTs). 2 RCTs (pooled) showed no difference between elastic and inelastic (short-stretch) outer bandages at 1 month, but 1 RCT showed a benefit for the elastic outer bandage at 1 year (RBI $248 \%$, CI 29\% to $951 \%$;
NNT 3, CI 2 to 12). 1 RCT showed greater healing with the 4LB than with a 2-component system at 6 months (RBI 79\%, CI $33 \%$ to $152 \%$; NNT 3, CI 2 to 6). 3-component compression systems (4 RCTs). In 3 RCTs, 3-component compression systems with a middle elastic bandage were compared with those with an inelastic middle bandage; pooled results for 2 showed a benefit for the elastic middle bandage at 3-4 months (RBI $83 \%$, CI $26 \%$ to $167 \%$; NNT 4, CI 3 to 9). The fourth RCT compared different 3-component systems and found no differences. 4-component compression systems: different versions of the $4 L B$ ( $3 R C T$ s). No differences were found among different 4LBs. $4 L B v$ multicomponent systems that included an inelastic bandage (6 RCTs). Pooled results adjusted for baseline ulcer area and other covariates showed a benefit for healing with the 4LB (4 RCTs, relative risk reduction [RRR] 12\%, CI $2 \%$ to $22 \%$; NNT 13, CI 7 to 91$)$. 4 LB v compression systems with paste bandage (Unna's boot) as base (5 RCTs). Groups did not differ for complete healing. Adjustable compression boots $v$ compression bandage systems (2RCTs). Groups did not differ for complete healing. Compression stockings/tubular devices $v$ compression bandage systems (6 RCTs). No differences were seen between single-layer stockings and paste bandages (2 RCTs). More patients achieved healing with 2-layer stockings than with the short-stretch bandage at 3 months (2 RCTs, RBI $72 \%$, CI $14 \%$ to $158 \%$; NNT 6 , CI 3 to 17$)$. 2 RCTs compared tubular compression devices with compression bandage systems; 1 showed that more cumulative patients healed in the tubular-compression group than in the compression-bandage group ( $93 \%$ v 51\%, p <0.001).

\section{CONCLUSIONS}

Patients with venous ulcers heal more quickly with compression than without. Multicomponent systems lead to better healing than single-component compression. Alternative systems with 2 or 3 components seem more effective when an elastic bandage is included. Different versions of the 4-layer bandage achieve similar healing rates.

\section{ABSTRACTED FROM}

O'Meara S, Cullum NA, Nelson EA. Compression for venous leg ulcers. Cochrane Database Syst Rev 2009;(1):CD000265.

Correspondence to: Dr S O'Meara, University of York, York, UK; smo4@york.ac.uk Source of funding: National Institute for Health Research, UK.

- Clinical impact ratings: General/internal medicine 6/7; Haematology (except oncology) 5/7; Wound care 6/7 $\prod$ he timely review by O'Meara at al evaluates comprehensively the current evidence base for use of compression therapy in the management of venous ulceration. It supports the use of compression as the "gold standard" for uncomplicated venous leg ulcers and the finding that multicomponent systems containing elastic bandages are more effective than those composed mainly of inelastic constituents. Importantly, it shows improvement in recent years in the methodological quality of included RCTs, which adds strength to the conclusions of the review. Consequently, research for management of venous ulcers must now surely shift to implementation strategies. This should focus on education and training of professionals, and improvement of healthcare resources that can support implementation. With an estimated 490000 to 1.3 million European Union citizens having leg ulceration (all types), there are examples of best practice that could guide implementation and further improve patient outcomes. ${ }^{12}$

This review identifies the need for future research to incorporate cost-effectiveness and quality of life measures within protocols. In the current constrained economic environment, such requests should be supported as this may influence not only current service delivery but future resource allocation for management of venous leg ulcers. The authors have provided a valuable resource of current evidence that will be of value to clinicians, policy makers, governing bodies, and patients alike.

\section{Georgina Gethin, RGN, PhD, FFNMRCSI}

Royal College of Surgeons in Ireland

Dublin, Ireland

1. Posnett J, Gottrup F, Lundgren $\mathrm{H}$, et al. The resource impact of wounds on health-care providers in Europe. $J$ Wound Care 2009;18:154-61.

2. Clarke-Moloney M, Keane N, Kavanagh E. Changes in leg ulcer management practice following training in an Irish community setting. J Wound Care 2008;17: $116,118-21$. 